BULLETIN OF THE

AMERICAN MATHEMATICAL SOCIETY

Volume 80, Number 2, March 1974

\title{
FROBENIUS RECIPROCITY OF DIFFERENTIABLE REPRESENTATIONS
}

\author{
BY JOHAN F. AARNES
}

Communicated by François Treves, July 10, 1973

\begin{abstract}
In this note we give the construction of the adjoint and the coadjoint of the restriction functor in the category of differentiable $G$-modules, where $G$ is a Lie group.
\end{abstract}

1. Introduction. Let $G$ be a Lie group, countable at infinity. A continuous representation $\lambda$ of $G$ in a complete locally convex space $E$ is differentiable if for each $a \in E$ the map $\hat{a}: x \rightarrow \lambda(x) a$ of $G$ into $E$ is $C^{\infty}$, and if the injection $a \rightarrow \hat{a}$ of $E$ into $C^{\infty}(G, E)$ is a topological homeomorphism [8]. We then say that $E$ is a differentiable $G$-module.

There is a natural way of associating a differentiable representation to any continuous, in particular unitary, representation of $G$. In fact, let $\rho$ be a continuous representation of $G$ on a complete locally convex space $F$. Let $F_{\infty}=\left\{a \in F: \hat{a} \in C^{\infty}(G, F)\right\}$. Then $F_{\infty}$ is a dense $\rho$-invariant linear subspace of $F$. The injection $a \rightarrow \hat{a}$ sends $F_{\infty}$ onto a closed subspace of $C^{\infty}(G, F)$. When $F_{\infty}$ is equipped with the relative topology of $C^{\infty}(G, F)$ it becomes a complete locally convex space, and the corresponding subrepresentation $\lambda_{\infty}$ of $\lambda$ on $F_{\infty}$ is differentiable. If $\lambda$ is topologically irreducible then $\lambda_{\infty}$ is topologically irreducible and conversely. For details and other basic facts concerning differentiable representations see [8].

The purpose of the present note is to show that the Frobenius reciprocity theorem is valid in the category of differentiable $G$-modules. The history of the Frobenius reciprocity theorem is long and interesting. For some recent developments the reader is referred to the work of Bruhat [1], Moore [4], Rieffel [5] and Rigelhof [6]. In particular Rigelhof succeeded in constructing an adjoint and a coadjoint for the restriction functor in the category of continuous (locally convex) $G$-modules.

2. Construction of the adjoint and the coadjoint. Let $K$ be a closed subgroup of $G$, and let $F$ be a differentiable $G$-module. The restriction $F \rightarrow F_{K}$ is a functor from the category of differentiable $G$-modules to the category of differentiable $K$-modules.

Let $E$ be a differentiable $K$-module and let $\pi$ be the corresponding representation.

(1) Coadjoint functor. Let $\mathscr{E}^{\prime}=\mathscr{E}^{\prime}(G)$ denote the space of distributions

AMS (MOS) subject classifications (1970). Primary 22D12, 22D30, 22E45; Secondary $43 \mathrm{~A} 65$.

Key words and phrases. Frobenius reciprocity, differentiable representations, adjoint functor, coadjoint functor. 
with compact support on $G$, equipped with the strong topology as the dual of $C^{\infty}(G)$.

Let $E^{G}$ denote the space of all continuous $K$-linear maps of $\mathscr{E}^{\prime}$ into $E$, i.e., $E^{G}=\operatorname{Hom}_{K}\left(\mathscr{E}^{\prime}, E\right)$ and $m \in E^{G}$ iff

$$
m(k S)=k m(S) ; \quad S \in \mathscr{E}^{\prime}, k \in K .
$$

Here $(k S)(f)=S(f k)$, where $(f k)(x)=f(k x), f \in C^{\infty}(G), x \in G . E^{G}$ is given the topology of uniform convergence on compact sets and is a complete locally convex space. We define the induced representation $\pi^{G}$ of $G$ on $E^{G}$ by

$$
\left[\pi^{G}(x) m\right](S)=m(S x)
$$

Proposition 1. $\pi^{G}$ is a differentiable representation of $G$ on $E^{G}$.

(2) Adjoint functor. A bilinear map $\omega: \mathscr{E}^{\prime} \times E \rightarrow H, H$ a locally convex space is $K$-balanced if $\omega(S k, a)=\omega(S, k a)$ for all $S \in \mathscr{E}^{\prime}, k \in K, a \in E$. (We let $K$ act to the right on $\mathscr{E}^{\prime}$ here and write $k a$ for $\pi(k) a$.) Let $B_{K}\left(\mathscr{E}^{\prime}, E\right)$ denote the space of all $K$-balanced bilinear maps of $\mathscr{E}^{\prime} \times E$ into $C$. Let $\chi: \mathscr{E}^{\prime} \times E \rightarrow B_{K}\left(\mathscr{E}^{\prime}, E\right)^{*}$ be the canonical map:

$$
\chi(S, a) b=b(S, a) ; \quad b \in B_{K}\left(\mathscr{E}^{\prime}, E\right) .
$$

$\chi$ is $K$-balanced and bilinear. We let $\mathscr{E}^{\prime} \otimes_{K} E$ denote the linear span of the range of $\chi$. Typical elements of $\mathscr{E}^{\prime} \otimes_{K} E$ will be written $\sum_{i=1}^{n} S_{i} \otimes a_{i}$. We give $\mathscr{E}^{\prime} \otimes_{K} E$ the inductive tensor product topology with respect to the family of bounded subsets of $\mathscr{E}^{\prime}$ and $E$ [2]. Let ${ }^{a} E$ be the completion of $\mathscr{E}^{\prime} \otimes_{K} E$ with respect to this topology. We define the representation ${ }^{a} \pi$ of $G$ on ${ }^{a} E$ by

$$
{ }^{a} \pi(x)(S \otimes a)=x S \otimes a .
$$

This construction is similar to the one given in [5] and [6].

Proposition 2. ${ }^{a} \pi$ is a differentiable representation of $G$ on ${ }^{a} E$.

3. Main result. Preserve the notation and assumptions above.

THEOREM 1. $E \rightarrow{ }^{G} E$ is the adjoint and $E \rightarrow E^{G}$ is the coadjoint functor of the restriction functor $F \rightarrow F_{K}$, i.e., there are natural isomorphisms (in the sense of category theory):

$$
\begin{aligned}
& \operatorname{Hom}_{G}\left({ }^{G} E, F\right) \cong \operatorname{Hom}_{K}\left(E, F_{K}\right) ; \\
& \operatorname{Hom}_{G}\left(F, E^{G}\right) \cong \operatorname{Hom}_{K}\left(F_{K}, E\right) .
\end{aligned}
$$

Moreover, the adjoint and coadjoint are unique to within equivalence of differentiable G-modules.

REMARK. The construction of the isomorphism in (3.1) rests upon the preliminary result that $\omega:(S, a) \rightarrow \lambda(S) a$ is a hypocontinuous bilinear map of $\mathscr{E}^{\prime} \times F$ into $F$. Here $\lambda(S)$ denotes the distribution form of the 
representation $\lambda$. $\omega$ is also $K$-balanced, and therefore any $A \in \operatorname{Hom}_{K}\left(E, F_{k}\right)$ determines a continuous linear map $A^{\prime}: \mathscr{E}^{\prime} \otimes_{K} E \rightarrow F$ such that

$$
A^{\prime} \sum S_{i} \otimes a_{i}=\sum \lambda\left(S_{i}\right) A a_{i}=\sum \omega\left(S_{i}, A a_{i}\right) \text {. }
$$

The map $A \rightarrow A^{\prime}$ defines (3.1).

For (3.2) let $A \in \operatorname{Hom}_{K}\left(F_{K}, E\right)$, and let $a \in F$. Define $\left(A^{\prime} a\right)(S)=$ $A \lambda(S) a$. Then $A^{\prime} a$ belongs to $\operatorname{Hom}_{K}\left(\mathscr{E}^{\prime}, E\right)=E^{G}$ and $A^{\prime}: a \rightarrow A^{\prime} a$ belongs to $\operatorname{Hom}_{G}\left(F, E^{G}\right)$. The map $A \rightarrow A^{\prime}$ defines (3.2).

Finally, both (3.1) and (3.2) are topological isomorphisms (with respect to standard topologies).

4. Realizations. In this section we give alternative descriptions of the $G$-modules $E^{G}$ and ${ }^{G} E$. As may be expected $E^{G}$ may be realized as a space of $E$-valued $C^{\infty}$-functions. Let $C_{K}^{\infty}(G, E)$ denote the space of $C^{\infty}$. functions $f: G \rightarrow E$ satisfying

$$
f(k x)=\pi(k) f(x) ; \quad k \in K, x \in G .
$$

We give $C_{K}^{\infty}(G, E)$ the relative topology from $C^{\infty}(G, E)$, and let $G$ act as the right regular representation on $C_{K}^{\infty}(G, E)$. This makes $C_{K}^{\infty}(G, E)$ into a differentiable $G$-module, and we have

Proposition 3. The differentiable G-modules $E^{G}$ and $C_{K}^{\infty}(G, E)$ are equivalent.

The proof is more or less straightforward, based on the isomorphisms $\operatorname{Hom}\left(\mathscr{E}^{\prime}, E\right) \cong C^{\infty}(G) \hat{\otimes} E \cong C^{\infty}(G, E)\left(C^{\infty}(G)\right.$ is a reflexive nuclear space; see [7].)

It does not appear possible to realize ${ }^{G} E$ as a space of functions. There is, however, another representation of ${ }^{G} E$, in a particular case, which throws some light on the connection between ${ }^{G} E$ and $E^{G}$.

Let $C_{c}^{\infty}(G)$ denote the space of complex-valued $C^{\infty}$-functions on $G$ with compact support, equipped with the usual topology [7], [8]. Let $\operatorname{Hom}_{K}^{0}\left(C_{c}^{\infty}(G), E\right)$ be the space of all continuous linear maps $m: C_{c}^{\infty}(G) \rightarrow E$ which satisfy

$$
\begin{gathered}
\text { supp } m \subseteq C K \text { for some compact subset } C \text { of } G ; \\
m(k \varphi)=\delta(k)^{-1} k m(\varphi) \text { where } k \in K, \varphi \in C_{c}^{\infty}(G), \text { and } \\
\delta \text { is the modular function of } K .
\end{gathered}
$$

We topologize $\operatorname{Hom}_{K}^{0}\left(C_{c}^{\infty}(G), E\right)$ as follows. For each compact set $C$, let $\operatorname{Hom}_{K}^{C}\left(C_{c}^{\infty}(G), E\right)$ be the subspace of those $m$ 's that have their support in $C K$. We give this space the relative topology as a subspace of $\operatorname{Hom}\left(C_{c}^{\infty}(G), E\right) . \operatorname{Hom}_{K}^{0}\left(C_{c}^{\infty}(G), E\right)$ is then given the inductive limit topology from the family of spaces $\operatorname{Hom}_{K}^{C}\left(C_{c}^{\infty}(G), E\right)$ as $C$ runs through the collection of compact subsets of $G$. We make $\operatorname{Hom}_{K}^{0}\left(C_{c}^{\infty}(G), E\right)$ into 
a differentiable $G$-module by the action

$$
(x m) \varphi=m(\varphi x) ; \quad \varphi \in C_{c}^{\infty}(G), x \in G .
$$

Proposition 4. If $E$ is the dual of a reflexive Fréchet space then the differentiable G-modules ${ }^{G} E$ and $\operatorname{Hom}_{K}^{0}\left(C_{c}^{\infty}(G), E\right)$ are equivalent.

The proof of this result rests upon another proposition, stated below. Let $\operatorname{Hom}^{0}\left(C_{c}^{\infty}(G), E\right)$ be the space of all continuous linear maps $m$ : $C_{c}^{\infty}(G) \rightarrow E$ with compact support. We equip this space with the natural inductive topology. Let $K$ act to the right on the space by

$$
(m k) \varphi=\delta(k) k^{-1} m(k \varphi) \text {. }
$$

Then define

$$
m^{\#} \varphi=\int_{K}(m k) \varphi \mathrm{d} k ; \quad k \in K, \varphi \in C_{c}^{\infty}(G) .
$$

Proposition 5. The map $m \rightarrow m^{\#}$ is linear, continuous, and open of $\operatorname{Hom}^{0}\left(C_{c}^{\infty}(G), E\right)$ onto $\operatorname{Hom}_{K}^{0}\left(C_{c}^{\infty}(G), E\right)$.

Remark. This result is true when $E$ is a complete locally convex space.

5. Concluding remarks. Bruhat [1] has given another definition of differentiably induced representations, and has given a version of the Frobenius reciprocity theorem in terms of intertwining forms. Full proofs of the results above, a discussion of the relationship to Bruhat's work, and other results (inducing in stages, etc.) will be given elsewhere.

\section{REFERENCES}

1. F. Bruhat, Sur les représentations induites des groupes de Lie, Bull. Soc. Math. France 84 (1956), 97-205. MR 18, 907.

2. A. Grothendieck, Produits tensoriels topologique et espaces nucléaires, Mem. Amer. Math. Soc. No. 16 (1955). MR 17, 763.

3. S. Mac Lane, Categorical algebra, Bull. Amer. Math. Soc. 71 (1965), 40-106. MR 30 \#2053.

4. C. C. Moore, On the Frobenius reciprocity theorem for locally compact groups, Pacific J. Math. 12 (1962), 359-365. MR 25 \#5134.

5. M. A. Rieffel, Induced Banach representations of Banach algebras and locally compact groups, J. Functional Analysis 1 (1967), 443-491. MR 36 \#6544.

6. R. Rigelhof, Induced representations of locally compact groups, Acta Math. 125 (1970), 155-187. MR 43 \#7550.

7. F. Treves, Topological vector spaces, distributions and kernels, Academic Press, New York, 1967. MR 37 \#726.

8. G. Warner, Harmonic analysis on semi-simple Lie groups. I, Springer-Verlag, New York and Berlin, 1972.

Department of Mathematics, University of Colorado, Boulder, Colorado 80302

Current address: University of Trondheim, Trondheim, Norway 